\title{
Extending scaled-interaction adaptive-partitioning QM/MM to covalently bonded systems
}

\author{
Zeng-hui Yang,,$\dot{\dagger}$, \\ Microsystem and Terahertz Research Center, China Academy of Engineering Physics, Chengdu, \\ China 610200, and Institute of Electronic Engineering, China Academy of Engineering Physics, \\ Mianyang, China 621000 \\ E-mail: yangzenghui@mtrc.ac.cn
}

\section{Abstract}

Quantum mechanics/molecular mechanics (QM/MM) is the method of choice for atomistic simulation$\mathrm{s}$ of large systems that can be partitioned into active and environmental regions. Adaptivepartitioning (AP) methods extend the applicability of $\mathrm{QM} / \mathrm{MM}$, allowing active zones to change during the simulation. AP methods achieve continuous potential energy surface (PES) by introducing buffer regions in which atoms have both $\mathrm{QM}$ and MM characters. Most of the existing AP-QM/MM methods require multiple QM calculations per time step, which can be expensive for systems with many atoms in buffer regions. Although one can lower the computational cost by grouping atoms into fragments, this may not be possible for all systems, especially for applications in covalent solids. The SISPA method [J. Chem. Theory Comput. 2017, 13, 2342] differs from other AP-QM/MM methods by only requiring one QM calculation per time step, but it has the flaw that the QM charge density and wavefunction near the buffer/MM boundary tend to those of isolated atoms/fragments. Besides, regular QM/MM methods for treating covalent bonds cut by the QM/MM boundary are incompatible with SISPA. Due to these flaws, SISPA in its original form

\footnotetext{
${ }^{*}$ To whom correspondence should be addressed

${ }^{\dagger}$ Microsystem and Terahertz Research Center, China Academy of Engineering Physics, Chengdu, China 610200

Institute of Electronic Engineering, China Academy of Engineering Physics, Mianyang, China 621000
}

cannot treat covalently bonded systems properly. In this work, we show that a simple modification to the SISPA method improves the treatment of covalently bonded systems. We also study the effect of correcting the charge density in SISPA by developing a density-corrected pre-scaled algorithm. We demonstrate our methods with simple molecules and bulk solids.

\section{Introduction}

Quantum mechanics/molecular mechanics (QM/MM) ${ }^{1-7}$ methods combine the accuracy of QM methods and the computational efficiency of MM method$\mathrm{s}$, allowing accurate atomistic simulation of large systems. QM/MM methods partition the system into QM and MM subsystems corresponding to the active and the environmental regions. Such a partition is predetermined in regular $\mathrm{QM} / \mathrm{MM}$, which is unfavorable when active regions are not stationary or liable to change during the simulation, such as in solution systems ${ }^{8-10}$ or transport processes. ${ }^{11-13}$ Adaptive-partitioning (AP) $\mathrm{QM} / \mathrm{MM}^{6,7,14-23}$ addresses this problem by allowing the partitioning of the system to change during the simulation. Various criteria for partitioning the system on the fly have been developed, such as partition by distances to active sites, ${ }^{14,15,17,18,20,21}$ by number, ${ }^{24}$ by density, $, 25,26$ by stress, ${ }^{27}$ and by error indicator, ${ }^{23}$ with the first one being the most commonly used criterion. AP-QM/MM introduces buffer regions to remove discontinuities in the potential energy surface (PES) as the partitioning 
changes. Atoms in buffer regions have both QM and MM characters. In the following, we denote atoms in the QM, buffer, or MM regions as QM atoms, buffer atoms, or MM atoms respectively.

The first AP-QM/MM method by Rode et al. ${ }^{14}$ mixes the QM and MM forces on buffer atoms to ensure a smooth transition. The main flaw of such force-based methods is the potential energy being unavailable. Many of the more recent adaptive $\mathrm{QM} / \mathrm{MM}$ methods are energy-based, where the potential energy is obtained by mixing QM and MM potential energies of different partitions. ${ }^{17,18,20}$ In each partition, a selection of buffer atoms are treated as QM atoms and the others treated as MM atoms. These methods require more than one QM calculations per time step.

The permuted adaptive partitioning (PAP) ${ }^{17}$ is the most comprehensive energy-based APQM/MM method. It includes $2^{N^{\text {buf }}}$ partitions, where $N^{\text {buf }}$ is the number of buffer atoms. The computational cost of PAP becomes prohibitively high when there are more than a few buffer atoms. One can group atoms into fragments to reduce the number of partitions in some cases, such as treating solute molecules ${ }^{17}$ or differen$\mathrm{t}$ sections of a biological channel ${ }^{12}$ as a whole instead of as individual atoms. This needs a thorough understanding of the system under study, and a general grouping scheme that retains the key characteristics of the original system is yet to be found. Significant development has been made in developing AP-QM/MM methods with much fewer configurations, such as the sorted adaptive partitioning (SAP), ${ }^{17}$ difference-based adaptive solvation (DAS), ${ }^{18}$ size-consistent multipartitioning (SCMP), ${ }^{20}$ and so on. These methods only requires $N^{\text {buf }} \mathrm{QM}$ calculations per time step, still higher than that of regular QM/MM.

The scaled interaction single partition adaptive (SISPA) ${ }^{21}$ is an AP-QM/MM method that only require one QM calculation per time step. Instead of averaging over partitions, SISPA carries out one QM calculation in an averaged sense with scaled interactions. SISPA has a similar computational cost as regular QM/MM methods, making it a promising method for large systems. The scaled interactions lead to non-trivial changes, however, with the most obvious one being that the QM charge density and wavefunction tend to those of isolated atoms/fragments near the buffer/MM boundary. This might be acceptable for weaklybonded systems such as solutions, ${ }^{21}$ but the effects on covalently bonded systems remain to be checked. The covalent interactions between QM and MM subsystems are missing from SISPA as well.

Semiconductor devices form the foundation of modern electronics. Further development of semiconductor technologies requires atomistic understanding of the processes in semiconductor materials and devices. Many technological processes in the fabrication of semiconductors, such as ion implantation ${ }^{28-30}$ and migration and reaction of defects or dopant atoms, ${ }^{31-36}$ happen across a large distance and have obvious active sites, making them suitable for studies with AP-QM/MM simulations. AP-QM/MM methods with multiple QM calculations per time step can be expensive for such simulations. In this paper, we extend the SISPA method to covalently bonded systems by developing a simple modification to the scaling scheme of the SISPA, which scales the covalent and noncovalent interactions differently so that the covalent interactions between QM and MM representations of the atoms are included. To study the impact of correcting the QM charge density, we develop a correction algorithm while preserving the continuity of the PES. We demonstrate our methods with small molecules and bulk silicon.

\section{Method}

In this section, we first briefly review the PAP and SISPA methods. We then describe our modifications to the SISPA scaling scheme of interaction$\mathrm{s}$, and finish with the density-corrected pre-scaled QM algorithm.

\subsection{A brief review of PAP and SISPA}

We follow the common practice of partitioning the system into QM, buffer and MM regions by distances to the centers of active sites. ${ }^{14,15,17,18,20,21}$ These centers are pre-chosen atoms or positions in the system, and their associated QM and buffer regions are spherical and spherical shell shaped with pre-defined radii and thicknesses. Fig. 1 illustrates 
the partitioning of the system.

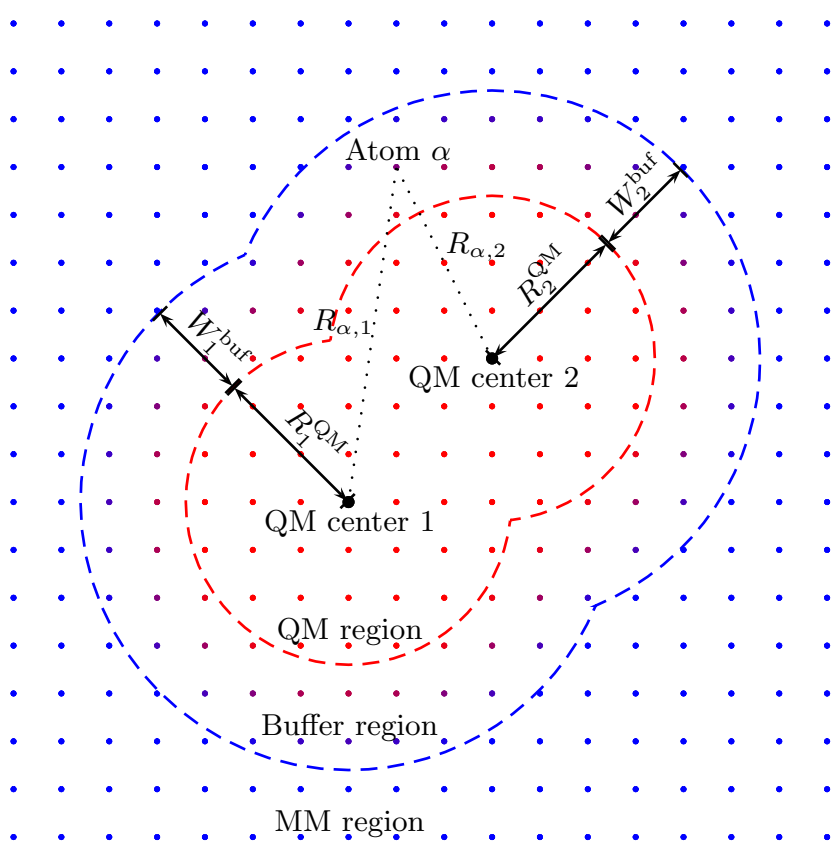

Figure 1: Illustration of the partitioning by distance scheme with dots representing atoms. The color of the dots represent scaling factors defined in Eq. (1), with blue being $\lambda=0$ and red being $\lambda=1 . R_{\zeta}^{\mathrm{QM}}$ and $W_{\zeta}^{\text {buf }}$ are the radius of the QM region and the thickness of the buffer region associated with center $\zeta$. and $R_{\alpha, \zeta}$ is the distance between atom $\alpha$ and center $\zeta$.

Buffer regions ensure a continuous PES as atoms move between QM and MM regions. Each atom in the system is assigned a scaling factor $\lambda$, signifying the QM character of the atom. $\lambda$ equals to 1 or 0 for QM or MM atoms, respectively. The scaling factor of a buffer atom $\alpha$ is: ${ }^{17}$

$$
\lambda_{\alpha}=1-\prod_{\zeta}\left(1-\lambda_{\alpha, \zeta}\right),
$$

where $\lambda_{\alpha, \zeta}$ denotes the scaling factor of $\alpha$ with respect to center $\zeta$ :

$$
\lambda_{\alpha, \zeta}=10 \tilde{\lambda}_{\alpha, \zeta}^{3}-15 \tilde{\lambda}_{\alpha, \zeta}^{4}+6 \tilde{\lambda}_{\alpha, \zeta}^{5}
$$

$\tilde{\lambda}_{\alpha, \zeta}$ in Eq. (2) is

$$
\begin{aligned}
\tilde{\lambda}_{\alpha, \zeta}=\frac{R_{\zeta}^{\mathrm{QM}}+W_{\zeta}^{\text {buf }}-R_{\alpha, \zeta}}{W_{\zeta}^{\text {buf }}} \theta\left(R_{\alpha, \zeta}-R_{\zeta}^{\mathrm{QM}}\right) \\
\quad \times \theta\left(R_{\zeta}^{\mathrm{QM}}+W_{\zeta}^{\text {buf }}-R_{\alpha, \zeta}\right)+\theta\left(R_{\zeta}^{\mathrm{QM}}-R_{\alpha, \zeta}\right),
\end{aligned}
$$

where $\theta$ is the Heaviside step function, $R_{\zeta}^{\mathrm{QM}}$ and $W_{\zeta}^{\text {buf }}$ are the radius of the QM region and the thickness of the buffer region of center $\zeta$, and $R_{\alpha, \zeta}$ is the distance between buffer atom $\alpha$ and center $\zeta$.

The PAP potential energy is a weighted sum of the potential energies of all partitions

$$
V^{\mathrm{PAP}}=\sum_{P} w_{P} V_{P}
$$

where $V_{P}$ is the regular QM/MM potential energy evaluated on partition $P$, and the weight of partition $P$ is

$$
w_{P}=\prod_{\alpha}^{\mathrm{QM}_{P}} \prod_{\beta}^{\mathrm{MM}_{P}} \lambda_{\alpha}\left(1-\lambda_{\beta}\right) .
$$

Many AP-QM/MM methods 17,18,20 improve the computational efficiency by only including some of the partitions in the summation.

Unlike the PAP method, a buffer atom in SISPA has both QM and MM representations at the same time. The QM and MM representations of the same atom do not interact with each other. Interactions between atoms are scaled in both the QM and MM calculations. The SISPA potential energy is

$$
V^{\mathrm{SISPA}}=V^{\mathrm{QM}, \mathrm{SISPA}}+V^{\mathrm{MM}, \mathrm{SISPA}},
$$

where $V^{\mathrm{QM}, \text { SISPA }}$ and $V^{\mathrm{MM} \text {,SISPA }}$ are the potential energies of scaled QM and MM calculations. Table 1 shows the scaling factors of interactions.

The scaled QM calculation lacks formal justification and can be thought as yielding an 'averaged' electronic structure. The SISPA method therefore trades rigorousness with higher computational efficiency. Unlike AP-QM/MM methods with multiple QM calculations per time step, the SISPA energy and forces have a non-linear dependency on the scaling factors, as can be seen with 
Table 1: Scaling factors of interactions of the SISPA method between atoms $\alpha$ and $\beta$. $\delta$ is the Kronecker $\delta$ notation.

\begin{tabular}{ccc}
\hline \hline & & QM calculation \\
\hline$\alpha$ & $\beta$ & Scaling \\
QM & QM & $\lambda_{\alpha} \lambda_{\beta}\left(1-\delta_{\alpha \beta}\right)+\delta_{\alpha \beta}$ \\
QM & MM & $\lambda_{\alpha}\left(1-\lambda_{\beta}\right)\left(1-\delta_{\alpha \beta}\right)$ \\
\hline \hline & & MM calculation \\
\hline MM & MM & $\left(1-\lambda_{\alpha}\right)\left(1-\lambda_{\beta}\right)\left(1-\delta_{\alpha \beta}\right)+\delta_{\alpha \beta}$ \\
\hline \hline
\end{tabular}

the simple model system in Appendix A.

Omitting the fragment corrections of the SISPA method, the zero of energy is defined by

$$
V_{0}^{\mathrm{SISPA}}=\sum_{\alpha}^{\mathrm{QM}} V_{0, \alpha}^{\mathrm{QM}}+\sum_{A}^{\mathrm{MM}} V_{0, A}^{\mathrm{MM}}
$$

where $V_{0, \alpha}^{\mathrm{QM}}$ and $V_{0, A}^{\mathrm{MM}}$ are the unscaled QM and M$M$ energies of isolated atom $\alpha$ and $A$ respectively. Since buffer atoms has both QM and MM representations, they are included in both the sums of Eq. (7).

The scaling factors in Table 1 assumes pairwise interactions. Complications arise in the implementation of SISPA when the QM or MM method contains interactions of more than two bodies. ${ }^{21}$ To avoid such complications, we use the densityfunctional tight-binding (DFTB) ${ }^{37-39}$ method and the charge-optimized many-body (COMB) potential $^{40-42}$ as the QM and MM methods in this paper, respectively. Refer to the supplemental material for details. ${ }^{43}$ This choice is made only to demonstrate the effect of scaling and to simplify the implementation. The compatibility of the QM and MM methods must be carefully tested in real applications.

\subsection{Treatment of covalent interactions}

For PAP and related methods, covalent bonds cut by the QM/MM boundary of a certain partition can be treated with link atoms ${ }^{44}$ or other techniques in regular QM/MM. ${ }^{3}$ The same cannot be done for SISPA due to scaled interactions. In this section, we present a simple modification to the SISPA scaling for treating covalent interactions between $\mathrm{QM}$ and MM representations of atoms, and we de- velop a method correcting the QM charge density in buffer regions.

\subsubsection{Scaled interaction for covalently bonded systems}

The SISPA method employs electrostatic embedding, ${ }^{2,45}$ so that scaled non-covalent interactions (such as electrostatic and dispersion) between QM and $\mathrm{MM}$ representations are included in the QM Hamiltonian. In the QM calculation, non-covalent interactions switches smoothly between their QM and MM descriptions. The resulting QM potential energy and forces would be reasonable if covalen$\mathrm{t}$ interactions are insignificant in the system, such as in solutions. ${ }^{21}$ If the covalent interaction is nonnegligible, however, SISPA would yield unphysical results. This is due to covalent interactions between QM and MM representations missing from both the QM and the MM calculations in SISPA.

In regular QM/MM methods, similar problems arise when the QM/MM boundary cut through covalent bonds. $^{2-4}$ A commonly used correction is to add extra link atoms to the QM calculation to represent the cut bond. ${ }^{2,46-49}$ Other methods such as capping potentials, ${ }^{50}$ effective fragment potentials, ${ }^{51}$ localized orbitals ${ }^{52-54}$ and so on have been proposed. SISPA with its scaled QM calculation is incompatible with these corrections, however, since all the covalent bonds involving buffer atoms can be seen as being partially 'cut' due to scaling.

We modify the SISPA method by scaling covalent and non-covalent interactions differently, so that the missing covalent interactions between QM and MM representations are included in the MM calculation. This can be seen as treating these covalent interactions with mechanical embedding. ${ }^{2,45}$ Table 2 lists the modified scaling factors of interactions (denoted as 'mod-SISPA' in the following). This modification requires that the M$M$ potential can be decomposed into covalent and non-covalent contributions. Since the scaling of the QM calculation does not change from SISPA, this modification does not solve the problem of QM charge density tending to that of isolated atoms near the buffer/MM boundary in SISPA. A part of covalent interaction is therefore still missing for mod-SISPA. We attempt to address this problem in the next section. 
Table 2: Modified scaling factors of interactions that distinguishes between covalent and other interactions between atoms $\alpha$ and $\beta$. $\delta$ is the Kronecker $\delta$ notation. ' $\mathrm{C}$ ' and ' $\mathrm{N}$ ' means covalent and non-covalent interactions respectively.

\begin{tabular}{cccc}
\hline \hline & \multicolumn{2}{c}{ QM calculation } \\
\hline$\alpha$ & $\beta$ & Type & Scaling \\
QM & QM & CN & $\lambda_{\alpha} \lambda_{\beta}\left(1-\delta_{\alpha \beta}\right)+\delta_{\alpha \beta}$ \\
QM & MM & N & $\lambda_{\alpha}\left(1-\lambda_{\beta}\right)\left(1-\delta_{\alpha \beta}\right)$ \\
\hline \hline \multicolumn{4}{c}{ MM calculation } \\
\hline MM & MM & $\mathrm{C}$ & $\left(1-\lambda_{\alpha} \lambda_{\beta}\right)\left(1-\delta_{\alpha \beta}\right)+\delta_{\alpha \beta}$ \\
MM & MM & $\mathrm{N}$ & $\left(1-\lambda_{\alpha}\right)\left(1-\lambda_{\beta}\right)\left(1-\delta_{\alpha \beta}\right)+\delta_{\alpha \beta}$ \\
\hline \hline
\end{tabular}

\subsubsection{Density-corrected pre-scaled algorithm for QM calculation}

Link atoms in regular QM/MM not only add back the missing covalent interaction between QM and MM atoms, but also corrects the QM charge density and the wavefunction near the QM/MM boundary. Without them, one obtain unphysical wavefunctions with dangling bonds at the QM/MM boundary. The SISPA method also suffers from unphysical QM charge density and wavefunction. In many cases, density-driven errors is the dominant error in density-functional ${ }^{55-57}$ QM calculations, ${ }^{58}$ and the accuracy can be improved by correcting the charge density. ${ }^{59}$ mod-SISPA of Table 2 compensates the missing QM-MM interaction with MM-MM covalent interaction, but does not solve the problem of unphysical charge density.

In the following, we describe a density-corrected pre-scaled (DCP) algorithm for studying the effect of correcting the charge density in SISPA-like APQM/MM methods. The buffer regions in SISPA ensure the continuity of both the charge density and the PES as atoms move between the QM and $\mathrm{MM}$ regions. We split these two purposes of buffer regions in DCP by introducing a secondary buffer region, so that the original buffer region (referred to as the primary buffer region in the following) only ensures the continuity of the PES, and the secondary buffer region ensures the continuity of the charge density.

We define the secondary buffer region of each center as a concentric spherical shell outside the corresponding primary buffer region. We assign pre-scaling factors of all atoms according to Eqs.
(1), (2) and (3), with the $R_{\zeta}^{\mathrm{QM}}$ and $W_{\zeta}^{\text {buf }}$ of Eq. (3) replaced by $R_{\zeta}^{\mathrm{QM}}+W_{\zeta}^{\mathrm{prim}}$ and $W_{\zeta}^{\mathrm{sec}}$, where $W_{\zeta}^{\mathrm{prim}}$ and $W_{\zeta}^{\mathrm{sec}}$ are the thicknesses of the primary and secondary buffer regions of center $\zeta$ respectively. Fig. 2 demonstrates the pre-scaling and scaling factors with an 1D atom chain.

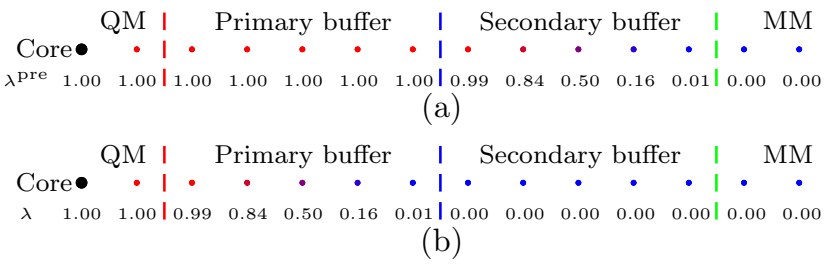

Figure 2: The pre-scaling and scaling factors of the DCP, illustrated with an $1 \mathrm{D}$ atom chain. (a) Pre-scaling factors in the self-consistent QM calculation of the first step. (b) Scaling factors in the non-self-consistent QM calculation of the second step. The pre-scaling and scaling factors are calculated with Eq. (2).

The DCP algorithm has two steps. In the first step, we carry out a self-consistent QM calculation with interactions scaled with the pre-scaling factors [Fig. 2(a)] and obtain the pre-scaled charge density and wavefunction. These are kept unchanged in the second step, and with them we carry out a non-self-consistent QM calculation with interactions scaled with scaling factors [Fig. 2(b)], which yields the QM potential energy and forces. The pre-scaled first step is the same as a SISPA QM calculation with a bigger QM region, and it ensures that the charge density and wavefunction change continuously as the QM subsystem change. The pre-scaling factors of QM and primary-buffer atoms equal to 1 , leading to a reasonable charge density inside the QM and primary buffer regions. Although the charge density in the secondary buffer region would still tend to that of isolated atoms, the effect of having such unphysical charge density is countered by the vanishing scaling factors of the secondary buffer atoms. The DCP algorithm is applied on top of the mod-SISPA scaling factors, since it represents the another part of the missing covalent interaction.

The Hellmann-Feynman theorem does not hold for DCP. The QM forces therefore contain extra terms involving derivatives of the KS orbitals. To avoid direct calculation of these derivatives, ${ }^{60}$ we 
derive the $\mathrm{z}$-vector method ${ }^{61,62}$ for DCP. The extra computational cost of DCP comparing with SISPA is mainly composed of the cost of two matrix diagonalizations, one of dimension $N_{\text {basis }} \times N_{\text {basis }}$, the other of dimension $\left(N_{\text {occ }} \times N_{\text {virt }}\right) \times\left(N_{\text {occ }} \times N_{\text {virt }}\right)$, where $N_{\text {basis }}, N_{\text {occ }}$ and $N_{\text {virt }}$ are the number of basis functions, of occupied and virtual orbitals, respectively. The computational cost of DCP with SCC-DFTB as the QM method is higher due to its reliance on Mulliken-type partial charges. Refer to the supplemental material for details. ${ }^{43}$

\section{Results}

\subsection{Computational details}

We implement SISPA, mod-SISPA, DCP and the scaled COMB potential in the LAMMPS ${ }^{63,64}$ code. The scaled DFTB calculation is performed with a modified version of the DFTB $+{ }^{65}$ code.

The self-consistent charge (SCC) DFTB $^{38}$ extends the original DFTB method ${ }^{37}$ by including atomic partial charges, which greatly improved the accuracy and transferability of the method. The scaling of QM interactions in SISPA-like methods can lead to instabilities in SCC-DFTB, however, and we have to switch to the original DFTB method instead of the more accurate SCC-DFTB as the QM method in some of the following calculations.

As explained in the introduction, our motivation of developing AP-QM/MM methods is to study the dynamics of the technological processes in the manufacture of semiconductor devices. We therefore carry out test calculations on Silicon dimer$\mathrm{s}$, trimers and bulk solid. For these systems, we cannot assign fixed MM charges to atoms since all atoms are identical, and charge equilibration $(\mathrm{QEq})^{40,66}$ methods for MM would introduce extra QM forces that depend on the derivative of MM charges with respect to atom positions, which can be difficult to calculate. To avoid complications, we set the MM charges to zero in the following.

\subsection{Silicon dimer and trimer}

We use $\mathrm{Si}_{2}$ and $\mathrm{Si}_{3}$ molecules as model systems to study the effect of the scaling. Fig. 3 shows how

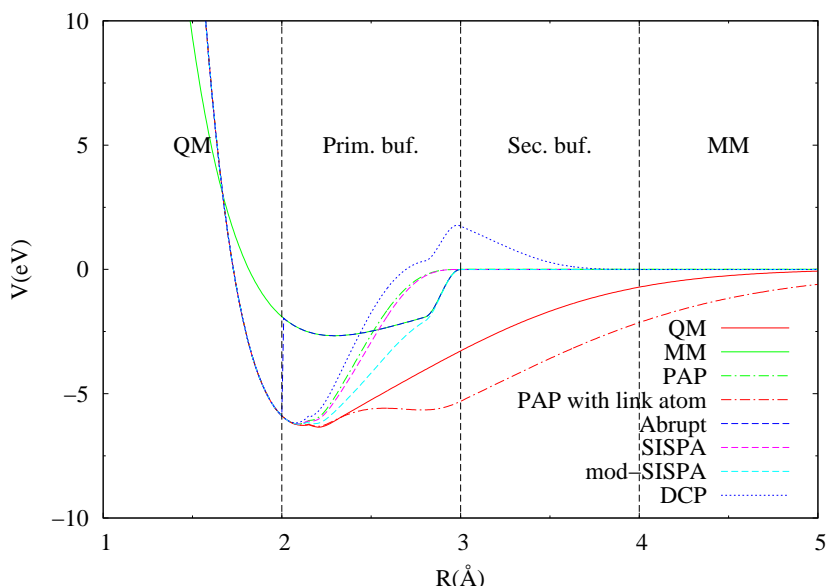

(a)

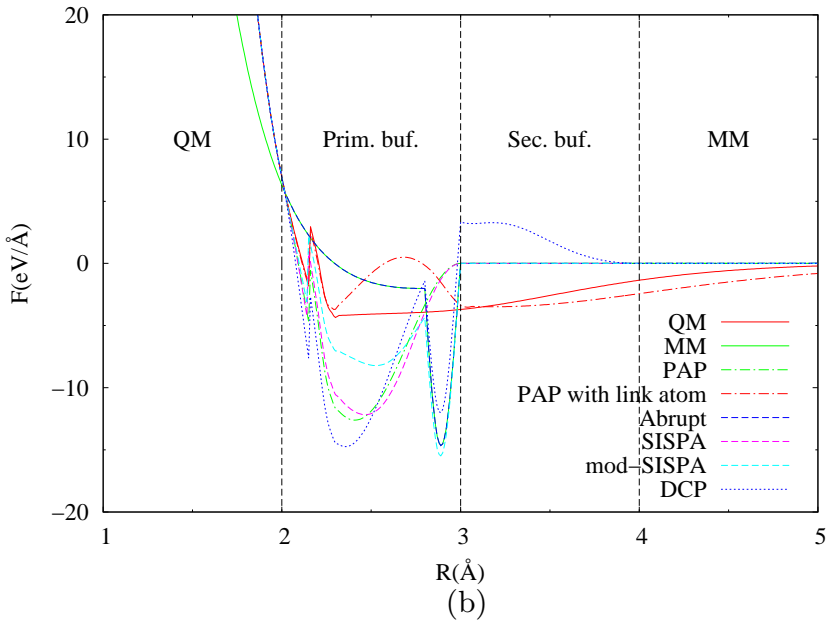

Figure 3: Potential energies and forces plotted versus bond length of $\mathrm{Si}_{2}$. One of the atom is the QM center, and the force on the other atom is plotted. The partition parameters are $R^{\mathrm{QM}}=2 \AA$, $W^{\text {prim }}=1.0 \AA$, and $W^{\mathrm{sec}}=1.0 \AA$. 'Abrupt' refers to the calculation without buffer region. 5,6 
the energy and force changes with the bond length of $\mathrm{Si}_{2}$. The potential energy curves of both modSISPA and DCP are continuous as expected. The potential well of SISPA is too narrow comparing with those of both the QM and MM, indicating the missing covalent interaction. The mod-SISPA of Table 2 compensates this with MM covalent interactions, leading to a wider potential well which is closer to both the QM and the MM potential wells. Since the PAP method is compatible with link atoms, ${ }^{44}$ we show the PAP curves both with and without hydrogen link atom for comparison, and the length of the link bond is scaled according to the length of the actual bond it represents. ${ }^{67} \mathrm{We}$ do not set a cutoff length for the link bond since it would lead to a discontinuous potential energy curve. PAP without link atom does not properly represent the covalent interaction, and its potential well has a very similar shape as that of SISPA in Fig. 3. Due to the inclusion of the QM-MM covalent interaction, PAP with link atom also leads to a wider potential well.

In Fig. 3, the interaction is overestimated when using link atoms to represent covalent bonds cut by the QM/MM boundary in PAP. This is due to the difference in the strength of the $\mathrm{Si}-\mathrm{Si}$ and $\mathrm{Si}-\mathrm{H}$ bonds. It should be noted that the length of the $\mathrm{Si}$ $\mathrm{H}$ link bond is determined by $\left(R_{\mathrm{Si}-\mathrm{Si}} / R_{\mathrm{Si}-\mathrm{Si}}^{\mathrm{eq}}\right) R_{\mathrm{Si}-\mathrm{H}}^{\mathrm{eq}}$ where $R^{\mathrm{eq}}$ is the equilibrium bond length, so the $\mathrm{Si}-\mathrm{H}$ interaction appears to be stronger than the $\mathrm{Si}$ $\mathrm{Si}$ interaction here. A better chosen partition parameter can alleviate this problem, as demonstrated in Fig. 4, but this may not be always possible in systems with more atoms. The mod-SISPA potential energy curve performs better in this aspect. It allows more freedom in the choice of partition parameters as it is always an interpolation between the QM and MM curves.

DCP appears to yield a even narrower potential well than that of SISPA, which is somewhat unexpected. Despite secondary-buffer atoms having vanishing scaling factors, the pre-scaling in DCP still has a prominent effect on the PES, which creates a barrier at the boundary of the primary and secondary buffer regions. Taking this barrier into account, the effective DCP potential well is wider than that of SISPA. Noticing the similarity of the shape of the DCP and mod-SISPA potential energy curves between $2.8 \AA$ and $3 \AA$, the effective

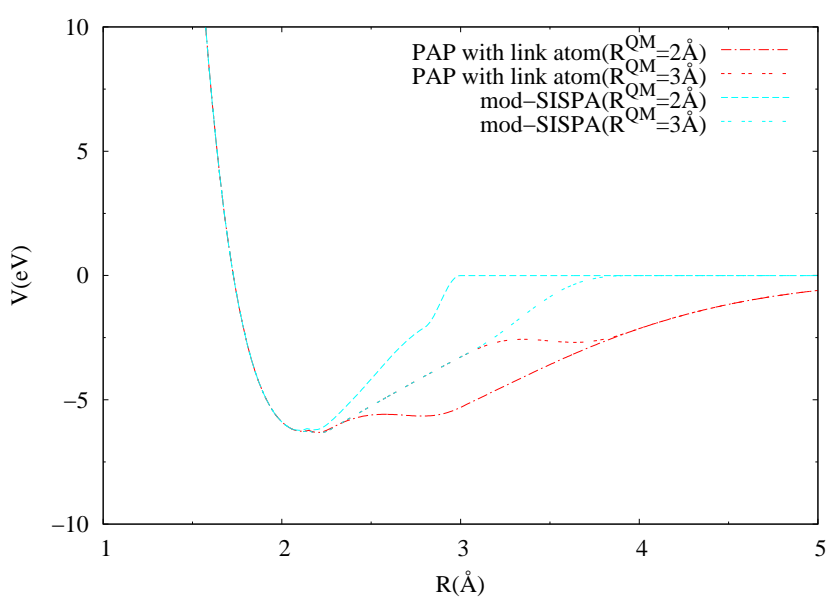

Figure 4: Potential energies versus atom distance in $\mathrm{Si}_{2}$ with different value of $R^{\mathrm{QM}}$. The system is the same as in Fig. 3. $W^{\text {buf }}=1.0 \AA$ for both $R^{\mathrm{QM}}=2.0 \AA$ and $R^{\mathrm{QM}}=3.0 \AA$.

DCP potential well is deeper than that of modSISPA when shifted down so that the curves between $2.8 \AA$ and $3 \AA$ are aligned. The difference between DCP and mod-SISPA signifies the missing part of the QM-MM covalent interaction due to the unphysical charge density. DCP curve for $R>3 \AA$ tends to the MM curve since the DCP PES is continuous, generating the artificial barrier at $3 \AA$. This artifact drives atoms away from the boundary of the buffer regions and would lead to distortions in the geometry, so DCP should not be used directly in AP-QM/MM simulations, and should only be used as a tool for analyzing the effect of charge density in SISPA-like methods.

The contribution of the scaling factors to the forces are unphysical and are responsible for geometry distortions. ${ }^{18,68,69}$ It has been proposed that such transition forces should be discarded directly, ${ }^{68}$ or an extra term should be added to the Hamiltonian to compensate the effect. ${ }^{17,18,69} \mathrm{We}$ plot the forces with and without transition forces in Fig. 5 for comparison, and find that the transition forces have a significant impact in SISPA-like methods as well. The forces of all AP-QM/MM methods becomes closer to QM forces with the transition forces removed. Due to the scaling of the interactions in SISPA-like methods, developing a correction to the Hamiltonian would be more difficult. In a real application of SISPA-like methods, it is more practical to discard the transition forces and couple the system to a thermostat. ${ }^{68}$ 


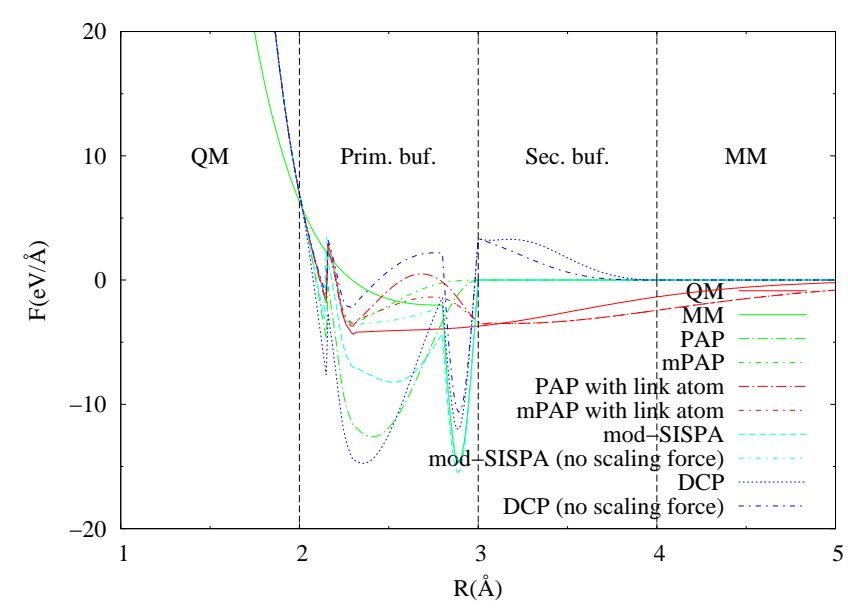

Figure 5: Comparison of the forces in Fig. 3(b) with those without forces depending on scaling and pre-scaling factors. The system is the same as in Fig. 3. mPAP $^{68}$ is PAP without transition forces due to derivatives of the weights.

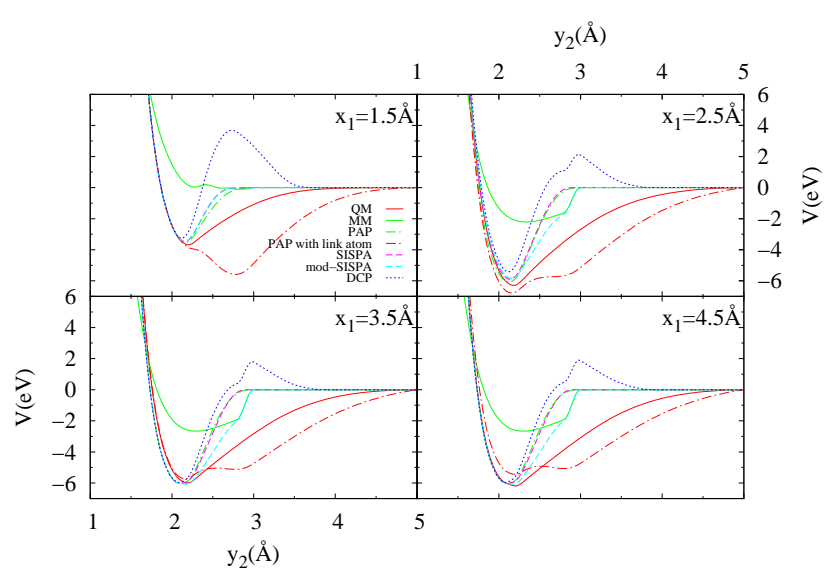

Figure 6: Potential energies of $\mathrm{Si}_{3}$, with one atom at the origin (atom 0 ) as the center and the other two atoms on the positive $x$ and $y$ axes respectively (atom 1 and 2). The partition parameters and calculation methods are the same as in Fig. 3. The curves are shifted so that they align at $y_{2}=5 \AA$ for easier comparison. The SCC-DFTB QM calculations of all curves are done with fractionally occupied orbitals corresponding to a temperature of $300 \mathrm{~K}$ to avoid numerical instabilities. For PAP with link atoms, we modified the QM code so that a link atom only interact with the atom it links to.
In the $\mathrm{Si}_{2}$ example, the center atom is always treated in QM, and the curves do not show the interaction between two buffer atoms. We plot the slices of the PES of three Si atoms in Fig. 6 to provide a better comparison. For the $x_{1}=1.5 \AA$ case, both atom 0 (center) and atom 1 are in the QM region, so correcting the charge density of atom 2 would change the QM interaction strengths of atom pair 0-2 and 1-2, leading to the artificial barrier of DCP being about twice the height than that of Fig. 3(b). When both atom 1 and 2 are in the buffer region, the effect of correcting the charge density is smaller, since the interaction is scaled by both atom's scaling factor. Again, we find that the PESs of PAP and SISPA have similar shapes, and mod-SISPA yields a better interpolation between QM and MM curves.

We only allow a link atom to interact with the corresponding QM atom of that bond to avoid double counting, but the shapes of the potential energy curves of PAP with link atoms vary more rapidly with $x_{1}$ than curves of other methods. This is due to that some of the partitions in PAP have $t$ wo hydrogen link atoms that represents the same $\mathrm{Si}$ atom, so the overestimation of the interaction in Fig. 3 is doubled here.

\section{3 bulk Si}

We apply the SISPA-like AP-QM/MM methods on a system of $4 \times 4 \times 4$ crystalline $S i$ supercell with 512 atoms. The lattice constant is fixed at $5.43 \AA$. Due to the large number of atoms involved, we are unable to carry out PAP calculations for comparison. For applications in covalent solids, it is usually impractical to reduce the computational cost by grouping atoms into fragments due to the system being highly uniform.

Fig. 7 shows the potential energy as an atom moves away from the QM center in bulk $\mathrm{Si}$. The potential energy curve of both QM and MM have the same general shape. The minima of the QM and MM potential energy curves are close to each other, indicating similar equilibrium bond lengths. For distances smaller than about $6 \AA$, the force on the moved atom points towards the center atom, showing the covalent interaction between them. The force switches to the opposite direction for larger distances as the interaction between the 


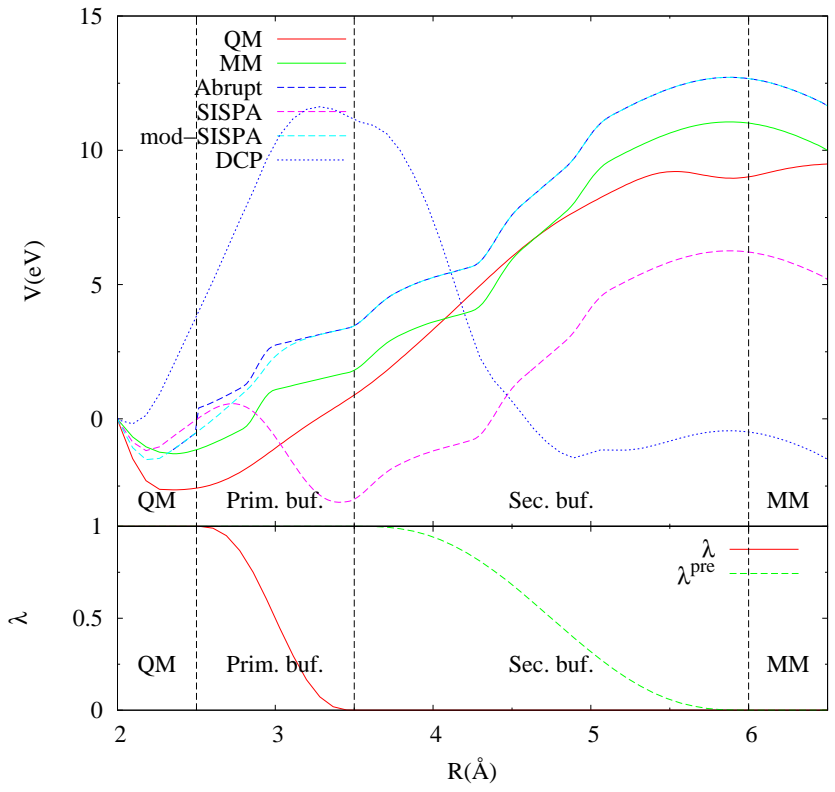

Figure 7: Potential energies and scaling factors as an atom in bulk Si moves away from the QM center. $R$ is the distance of the moved atom to the center atom. The moved atom is one of the nearest neighbors of the center. The partition parameters are $R^{\mathrm{QM}}=2.5 \AA, W^{\text {prim }}=W^{\text {buf }}=1 \AA$, and $W^{\text {sec }}=3 \AA$. The $\mathrm{QM}$ curve is obtained with only the $\Gamma$ point to be consistent with other calculations. The potential energy curves are shifted and aligned at $R=2 \AA$ for easier comparison. moved atom and another atom becomes stronger. Due to the missing covalent interaction between QM and MM representations, the SISPA potential energy curve is too low in the buffer region, generating the artificial potential well at about $3.5 \AA$, but the position of this potential well is determined by artificially chosen partition parameters and not directly related to the equilibrium bond lengths of both QM and MM. This would be problematic in molecular dynamics (MD) simulations since different partition parameters may lead to drastically different results. Correcting the charge density in DCP leads to an overcorrection. Instead of a potential well, DCP generates a barrier at the boundary of primary and secondary buffer regions, similar to the $\mathrm{Si}_{2}$ case in Fig. 3. mod-SISPA yields a better potential energy curve as it follows the general shape of both the QM and the MM curves. Even though the position of the minimum of the potential well is different from the QM and MM values, the difference is much smaller than that of SISPA, making it suitable for applications in bulk solids.

We carry out MD tests in the NVE ensemble with the 512 atom Si supercell, with the initial geometry and velocities obtained from a MD simulation with the COMB potential in the NVT ensemble in equilibration at $2000 \mathrm{~K}$. Fig. 8 shows the total energies during the simulations. We encounter severe convergence problem with SCCDFTB in QM and DCP calculations, and we have to switch to the original DFTB as the QM method for them. Unfortunately, we find that the original DFTB can lead to discontinuities with scaled interactions. The inset of Fig. 8 shows SISPA and mod-SISPA energy curves with the original DFT$\mathrm{B}$ as the QM method, and the total energy is not conserved due to this problem. The DCP algorith$m$ is less affected by this since the direct effect of the problem is on the charge density and orbitals of the pre-scaling step instead of on the energy.

The temperature variations of QM, MM, SISPA and mod-SISPA in Fig. 9 all have similar magnitudes. They are larger than that of PAP simulation with argon atoms ${ }^{17}$ due to the much stronger interaction in bulk $\mathrm{Si}$. The simulation with no buffer region ('Abrupt' curve in Fig. 9) shows artificial heating of the system, which is not present in other curves. 


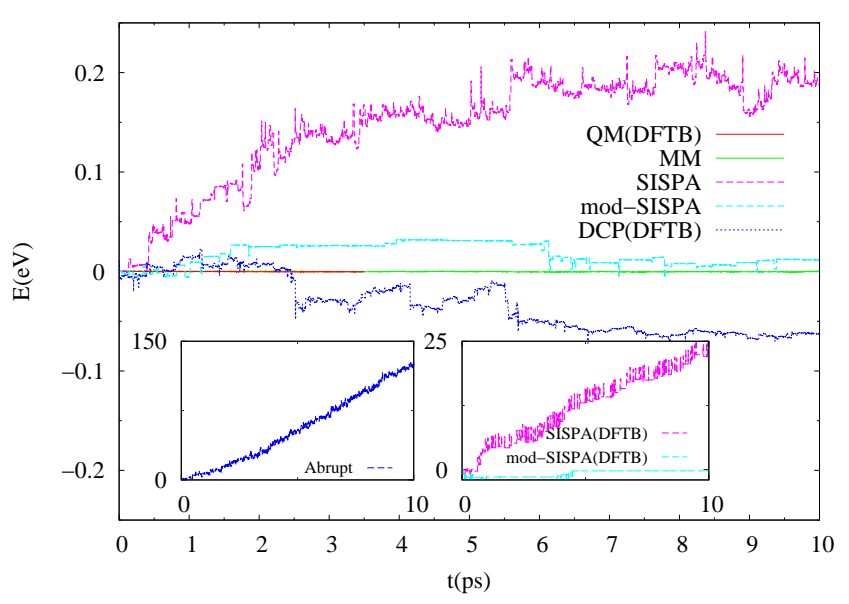

Figure 8: Total energies during MD simulations of bulk $\mathrm{Si}$ in the NVE ensemble with different methods. The time step is $0.1 \mathrm{fs}$. The QM calculation is run for $3.5 \mathrm{ps}$, and other calculations are run for 10 ps. The partition parameters are $R^{\mathrm{QM}}=2.5 \AA$, $W^{\text {prim }}=W^{\text {buf }}=1 \AA$, and $W^{\text {sec }}=1 \AA$. The curves are aligned at $\mathrm{t}=0$ for easier comparison. The $\mathrm{QM}$ calculation is carried out with only the $\Gamma$ point to be consistent with other calculations.

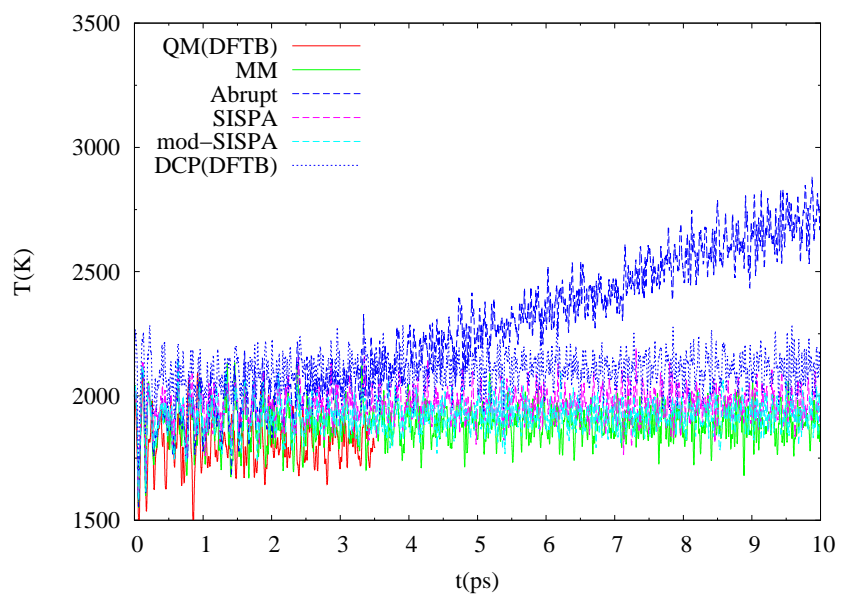

Figure 9: Temperature during MD simulations of bulk $\mathrm{Si}$ in the NVE ensemble with different methods. The setup of the simulations is the same as Fig. 8.
Fig. 10 illustrates the radial distribution function (RDF) of the QM center atom relative to all other atoms. The RDFs obtained from purely QM and purely MM simulations are similar to each other, but all the AP-QM/MM results are distorted. The RDF of SISPA in solutions shows that the first solvation shell is moved to the buffer boundary. ${ }^{21} \mathrm{We}$ find the opposite in bulk Si here, and the first shell of SISPA is moved towards the QM center. The shape of the RDFs can be roughly explained with the potential energy curves in Fig. 7. The first two peaks of the RDF of SISPA correspond to the two minima of the potential energy curve in Fig. 7, and the peak of the second shell is higher since its corresponding minima is lower in energy. Comparing with QM and MM RDFs, the peak of the second shell of SISPA is closer to the QM center. The interaction between the atoms in the first and second shells would then push the first shell towards the QM center, leading to the position of the first shell not exactly located at the first local minima of the curve in Fig. 7. DCP overestimates the density of the first shell, which is in accordance with the deep effective potential well in Fig. 7 due to the artificial barrier.

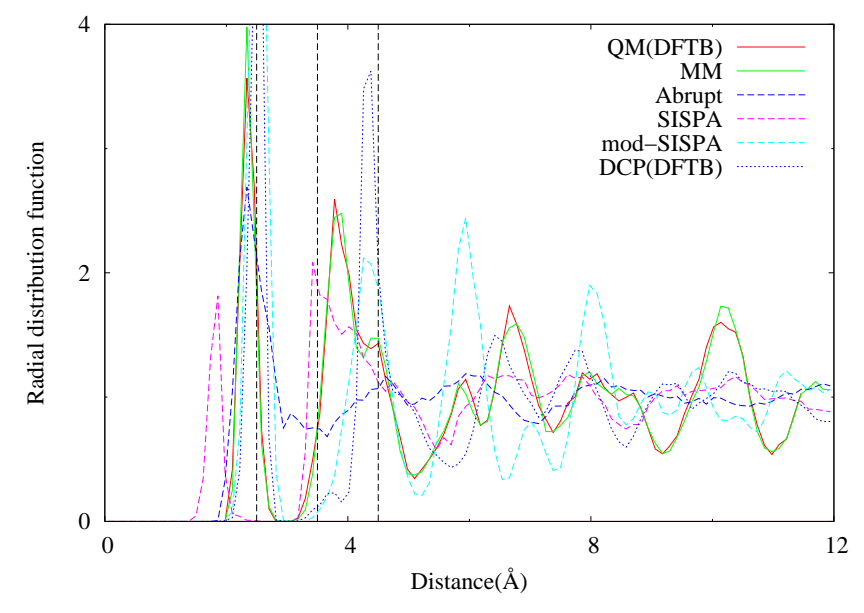

Figure 10: Radial distribution functions of the QM center atom relative to other atoms from MD simulations of bulk Si in the NVE ensemble. The setup of the simulation is the same as Fig. 8. The QM RDF is obtained from configurations sampled from 2 to $3.5 \mathrm{ps}$, and other RDFs are obtained from configurations sampled from 5 to 10 ps. The vertical dashed lines represent the boundary of QM, primary buffer and secondary buffer regions.

The RDF of mod-SISPA overestimates the den- 
sity of the first shell as well, and this cannot be explained with the curves in Fig. 7. It should be noted that Fig. 7 represents a highly simplified situation where only one atom moves, and the PES is a much more complicated object which may contain unexpected feature like this. We find that this problem is due to the transition forces, which also cause geometry distortions in other AP-QM/MM methods. ${ }^{18,68,69}$ Fig. 11 shows the RDFs calculated without transition forces, and mod-SISPA without transition forces no longer overestimate the density of the first shell. The RDFs no longer have a clear shell structure after removal of the transition forces, though mod-SISPA behaves better than SISPA in this aspect. A more sophisticated correction to transition forces might be needed to fully resolve this problem.

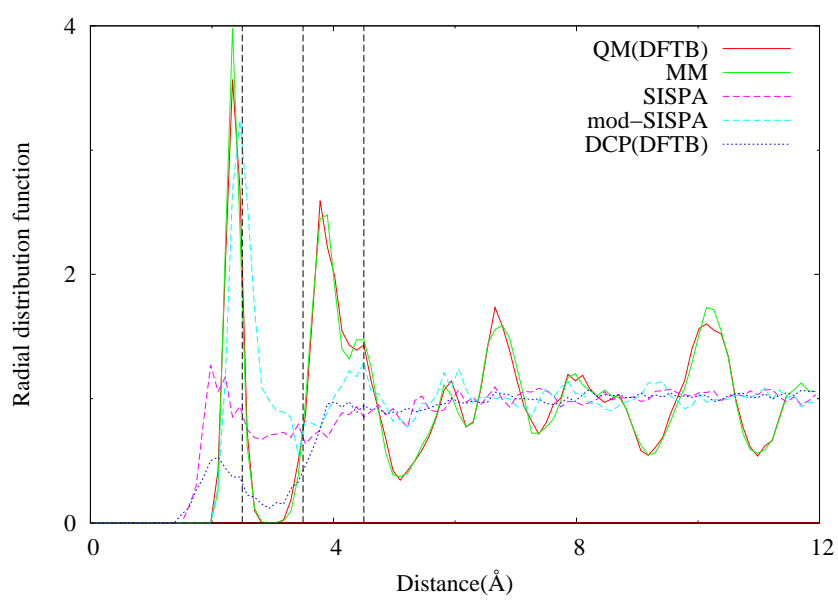

Figure 11: Radial distribution functions of SISPA, mod-SISPA and DCP without transition forces. The setup of the simulations is the same as Fig. 10 .

\section{Conclusion}

In this paper, we study methods extending the SISPA AP-QM/MM method to covalently bonded systems. While common energy-based APQM/MM methods are physically sound and would yield good results, their requirement of multiple QM calculations per time step can be overwhelming. Although grouping atoms into fragments reduces the number of partitions and the computational cost, it requires knowledge of the studied system beforehand, and there are also system- s where a reasonable grouping scheme may not exist, such as the bulk Si examples in this paper. The SISPA method achieves one QM calculation per time step with the cost of being less rigorous, since the meaning of the scaled QM calculation is vague. Despite this flaw, being able to do an AP-QM/MM calculation with a much smaller cost is quite attractive, since it opens up many possibilities for large systems that was inaccessible to common AP-QM/MM methods. Besides, AP-QM/MM methods with multiple QM calculations may contain partitions that lead to ill-defined QM calculations, especially when treating covalently bonded systems. It is therefore worthwhile to continue developing SISPA-like methods, even though there have been significant advancements in reducing the number of QM calculations in APQM/MM for specific systems. ${ }^{12,13}$

Various methods for treating covalent bonds cut by the QM/MM boundary have been developed for regular QM/MM. These methods can be applied to AP-QM/MM methods with multiple QM calculations per time step, since the calculation of each partition can be seem as a regular QM/MM calculation. Since these methods are incompatible with SISPA due to scaled interactions, we propose a new set of scaling factors (mod-SISPA) which compensates for the missing covalent interactions between QM and MM representations in the MM part of the SISPA method. We also develop the D$\mathrm{CP}$ algorithm that corrects the unphysical charge density of SISPA while keeping the PES continuous and smooth for studying the effect of the QM charge density in SISPA-like methods.

Tests in small model systems show that modSISPA yields a wider potential well than that of SISPA, indicating stronger interaction. The modSISPA potential energy can be better than that of PAP with link atoms in some aspects, since the latter may be negatively affected by the differences in the interaction strengths of the actual bond and the link bond, while the former is always close to the potential energies of QM or MM in different regions. We find that SISPA may yield a PES with an artificial minimum in bulk solids depending on the partition parameters, while mod-SISPA do not have this problem. The charge density and wavefunction tend to those of isolated atoms/fragments near the buffer/MM boundary in SISPA, and in- 
teractions between these atoms and other QM or buffer atoms are clearly underestimated. Correcting the charge density in DCP does lead to a deeper effective potential well in $\mathrm{Si}_{2}$, which seems to verify the underestimation of interactions in SISPA. The simple correction in DCP leads to significant artifacts in the PES, however, making it unsuitable for real applications. Similar to other AP-QM/MM methods, we also find that the transition forces due to scaling factors may lead to geometry distortions in SISPA-like methods as well.

\section{Acknowledgements}

Acknowledgement The author is supported by Science Challenge Project, No. TZ2016003, and by the National Natural Science Foundation of China grant No. 11804314.

\section{A The effect of scaling in a two- level model system}

The QM calculation in the SISPA method is carried out with scaled interactions. We demonstrate with a simple model that the SISPA energy and forces change in a non-linear fashion with scaling factors, unlike in PAP and other AP-QM/MM methods with multiple QM calculations per time step.

Here we consider a model system of two identical atoms ( $\mathrm{A}$ and $\mathrm{B}$ ) with one basis function on each $\left(\phi_{1}\right.$ and $\left.\phi_{2}\right)$ and ignore the MM potential. The distance between the atoms is $R$. The unscaled Hamiltonian and overlap matrices of the model system are

$$
\mathrm{H}=\left(\begin{array}{ll}
\varepsilon & v \\
v & \varepsilon
\end{array}\right)
$$

and

$$
\mathrm{S}=\left(\begin{array}{ll}
1 & s \\
s & 1
\end{array}\right)
$$

The corresponding orbitals and orbital energies are

$$
\begin{aligned}
\psi_{ \pm}(\vec{r}) & =\frac{1}{\sqrt{2}}\left(\phi_{1}(\vec{r}) \pm \phi_{2}(\vec{r})\right) \\
\varepsilon_{ \pm} & =\frac{\varepsilon \pm v}{1 \pm s}
\end{aligned}
$$

Assuming $\varepsilon_{+}<\varepsilon_{-}$, the DFTB bond energy is

$$
\begin{aligned}
E_{\mathrm{b}} & =E-2 \varepsilon \\
& =2\left\langle\psi_{+}|\mathrm{H}| \psi_{+}\right\rangle+E^{\mathrm{rep}}-2 \varepsilon \\
& =\frac{2(v-\varepsilon s)}{1+s}+E^{\mathrm{rep}},
\end{aligned}
$$

where $E^{\text {rep }}$ is the repulsive correction to the energy in DFTB. ${ }^{37,43}$ The forces on atoms A and B are

$$
\begin{aligned}
\vec{F}_{A} & =-\frac{\partial E_{b}}{\partial R} \nabla_{\vec{R}_{A}} R, \\
\vec{F}_{B} & =-\frac{\partial E_{b}}{\partial R} \nabla_{\vec{R}_{B}} R .
\end{aligned}
$$

Define $F=-\partial E_{b} / \partial R$, we have

$$
\begin{aligned}
F & =-\frac{\partial E_{\mathrm{b}}}{\partial R} \\
& =-2\left(\frac{\partial v}{\partial R}-\frac{\varepsilon+v}{1+s} \frac{\partial s}{\partial R}\right)-\frac{\partial E^{\mathrm{rep}}}{\partial R} .
\end{aligned}
$$

Choosing atom $\mathrm{A}$ as the center and atom $\mathrm{B}$ as a buffer atom, $\mathrm{H}$ and $\mathrm{S}$ becomes the following in SISPA:

$$
\mathrm{H}=\left(\begin{array}{cc}
\varepsilon & \lambda v \\
\lambda v & \varepsilon
\end{array}\right)
$$

and

$$
S=\left(\begin{array}{cc}
1 & \lambda s \\
\lambda s & 1
\end{array}\right),
$$

where $\lambda$ is the scaling factor of atom B. The SISPA bond energy and the magnitude of the forces of the model system are

$$
\begin{gathered}
E_{\mathrm{b}}^{\mathrm{SISPA}}=\frac{2 \lambda(v-\varepsilon s)}{1+\lambda s}+\lambda E^{\mathrm{rep}} \\
F^{\mathrm{SISPA}}=-\frac{2 \lambda}{1+\lambda s}\left(\frac{\partial v}{\partial R}-\frac{\varepsilon+\lambda v}{1+\lambda s} \frac{\partial s}{\partial R}\right)-\lambda \frac{\partial E^{\mathrm{rep}}}{\partial R} \\
-\frac{\partial E_{\mathrm{b}}^{\mathrm{SISPA}}}{\partial \lambda} \frac{\partial \lambda}{\partial R} .
\end{gathered}
$$

As a comparison, the bond energy and the magnitude of the forces with PAP are

$$
\begin{aligned}
E_{\mathrm{b}}^{\mathrm{PAP}} & =\lambda E+(1-\lambda) \varepsilon_{B}-2 \varepsilon \\
& =\lambda\left(\frac{2 v+\varepsilon(1-s)}{1+s}\right)-\varepsilon,
\end{aligned}
$$


and

$$
\begin{aligned}
F^{\mathrm{PAP}} & =-\frac{2 \lambda}{1+s}\left(\frac{\partial v}{\partial R}-\frac{\varepsilon+v}{1+s} \frac{\partial s}{\partial R}\right)-\lambda \frac{\partial E^{\mathrm{rep}}}{\partial R} \\
& -\frac{\partial E_{\mathrm{b}}^{\mathrm{PAP}}}{\partial \lambda} \frac{\partial \lambda}{\partial R} .
\end{aligned}
$$

Aside from the transition forces that are present in both SISPA and PAP, $E_{\mathrm{b}}^{\mathrm{PAP}}$ and $F^{\mathrm{PAP}}$ depend on $\lambda$ linearly, while $E_{\mathrm{b}}^{\text {SISPA }}$ and $F^{\text {SISPA }}$ has a non-linear dependence on $\lambda$.

\section{References}

(1) Warshel, A.; Levitt, M. J. Mol. Biol. 1976, 103, 227.

(2) Lin, H.; Truhlar, D. G. Theor. Chem. Acc. 2007, 117, 185.

(3) Senn, H. M.; Thiel, W. Angew. Chem. Int. Ed. 2009, 48, 1198.

(4) Bernstein, N.; Kermode, J. R.; Csányi, G. Rep. Prog. Phys. 2009, 72, 026501.

(5) Bulo, R. E.; Michel, C.; Fleurat-Lessard, P.; Sautet, P. J. Chem. Theory Comput. 2013, 9, 5567.

(6) Zheng, M.; Waller, M. P. WIREs Comput. Mol. Sci. 2016, 6, 369.

(7) Duster, A. W.; Wang, C.-H.; Garza, C. M.; Miller, D. E.; Lin, H. WIREs Comput. Mol. Sci. 2017, 7, e1310.

(8) Watanabe, H. C.; Banno, M.; Sakurai, M. Phys. Chem. Chem. Phys. 2016, 18, 7318.

(9) Watanabe, H. C.; Kubillus, M.; Kubař, T.; Stach, R.; Mizaikoff, B.; Ishikita, H. Phys. Chem. Chem. Phys. 2017, 19, 17985.

(10) Boereboom, J. M.; Fleurat-Lessard, P.; Bulo, R. E. J. Chem. Theory Comput. 2018, 14, 1841.

(11) Duster, A.; Garza, C.; Lin, H. Methods Enzymol. 2016, 577, 341.
(12) Duster, A. W.; Garza, C. M.; Aydintug, B. O.; Negussie, M. B.; Lin, H. J. Chem. Theory Comput. 2019, 15, 892.

(13) Duster, A. W.; Lin, H. J. Chem. Theory Comput. 2019, 15, 5794.

(14) Kerdcharoen, K. R., T. Liedl; Rode, B. M. Chem. Phys. 1996, 211, 313.

(15) Kerdcharoen, T.; Morokuma, K. Chem. Phys. Lett. 2002, 355, 257.

(16) Csányi, G.; Albaret, T.; Payne, M. C.; de Vita, A. Phys. Rev. Lett. 2004, 93, 175503.

(17) Heyden, A.; Lin, H.; Truhlar, D. G. J. Phys. Chem. B 2007, 111, 2231.

(18) Bulo, R. E.; Ensing, B.; Sikkema, J.; Visscher, L. J. Chem. Theory Comput. 2009, 5, 2212.

(19) Nielsen, S. O.; Bulo, R. E.; Moore, P. B.; Ensing, B. Phys. Chem. Chem. Phys. 2010, 12, 12401.

(20) Watanabe, H. C.; Kubař, T.; Elstner, M. J. Chem. Theory Comput. 2014, 10, 4242.

(21) Field, M. J. J. Chem. Theory Comput. 2017, 13, 2342.

(22) Watanabe, H. Molecules 2018, 23, 1882.

(23) Chen, H.; Liao, M.; Wang, H.; Wang, Y.; Zhang, L. Comput. Methods Appl. Mech. Eng. 2019, 354, 351.

(24) Takenaka, N.; Kitamura, Y.; Koyano, Y.; Nagaoka, M. Chem. Phys. Lett. 2012, 524, 56.

(25) Waller, M. P.; Kumbhar, S.; Yang, J. ChemPhysChem 2014, 15, 3218.

(26) Zheng, M.; Kuriappan, J. A.; Waller, M. P. Int. J. Quantum Chem. 2017, 117, 25336.

(27) Glukhova, O. E.; Savostyanov, G. V.; Slepchenkov, M. M. Procedia Materials Science 2014, 6, 256. 
(28) Chason, E.; Picraux, S. T.; Poate, J. M.; Borland, J. O.; Current, M.; Diaz de la Rubia, T.; Eaglesham, D. J.; Holland, O. W.; Law, M. E.; Magee, C. W.; Mayer, J. W.; Melngailis, J.; Tasch, A. F. J. Appl. Phys. 1997, 81, 6513 .

(29) Williams, J. S.; Elliman, R. G.; Ridgway, M. C.; Jagadish, C.; Ellingboe, S. L.; Goldberg, R.; Petravic, M.; Wong, W. C.; Dezhang, Z.; Nygren, E.; Svensson, B. G. Nucl. Instrum. Methods Phys. Res., Sect. B 1993, 80-81, 507.

(30) Borland, J. O.; Koelsch, R. Solid State Technol. 1993, 36, 28.

(31) Newman, R. C. Rep. Prog. Phys. 1982, 45, 1163.

(32) Clark, S. J.; Ackland, G. J. Phys. Rev. B 1997, $56,47$.

(33) Cowern, N. E. B.; van der Walle, G. F. A.; Zalm, P. C.; Oostra, D. J. Phys. Rev. Lett. 1992, 69, 116.

(34) Munro, L. J.; Wales, D. J. Phys. Rev. B 1999, 59,3969 .

(35) Gilmer, G. H.; Diaz de la Rubia, T.; Stock, D. M.; Jaraiz, M. Nucl. Instrum. Methods Phys. Res., Sect. B 1995, 102, 247.

(36) Alippi, P.; Colombo, L.; Ruggerone, P.; Sieck, A.; Seifert, G.; Frauenheim, T. Phys. Rev. B 2001, 64, 075207.

(37) Porezag, D.; Frauenheim, T.; Köhler, T.; Seifert, G.; Kaschner, R. Phys. Rev. B 1995, $51,12947$.

(38) Elstner, M.; Porezag, D.; Jungnickel, G.; Elsner, J.; Haugk, M.; Frauenheim, T.; Suhai, S.; Seifert, G. Phys. Rev. B 1998, 58, 7260.

(39) Frauenheim, T.; Seifert, G.; Elstner, M.; Hajnal, Z.; Jungnickel, G.; Porezag, D.; Suhai, S.; Scholz, R. Phys. Stat. Sol. (b) 2000, $217,41$.
(40) Yu, J.; Sinnott, S. B.; Phillpot, S. R. Phys. Rev. B 2007, 75, 085311.

(41) Shan, T.-R.; Devine, B. D.; Kemper, T. W.; Sinnott, S. B.; Phillpot, S. R. Phys. Rev. B 2010, 81, 125328.

(42) Liang, T.; Shan, T.-R.; Cheng, Y.-T.; Devine, B. D.; Noordhoek, M.; Li, Y.; Lu, Z.; Phillpot, S. R.; Sinnott, S. B. Mat. Sci. Eng. $R$ 2013, 74, 255.

(43) Refer to the supplemental material of this paper.

(44) Pezeshki, S.; Lin, H. J. Chem. Theory Comput. 2011, 7, 3625.

(45) Bakowies, D.; Thiel, W. J. Phys. Chem. 1996, 100, 10580.

(46) Field, M. J.; Bash, P. A.; Karplus, M. J. Comput. Chem. 1990, 11, 700.

(47) Maseras, F.; Morokuma, K. J. Comput. Chem. 1995, 16, 1170.

(48) Svensson, M.; Humbel, S.; Froese, R. D. J.; Matsubara, T.; Sieber, S.; Morokuma, K. J. Phys. Chem. 1996, 100, 19357.

(49) Das, D.; Eurenius, K. P.; Billings, E. M.; Sherwood, P.; Chatfield, D. C.; Hodošek, M.; Brooks, B. R. J. Chem. Phys. 2002, 117, 10534.

(50) DiLabio, G. A.; Wolkow, R. A.; Johnson, E. R. J. Chem. Phys. 2005, 122, 044708.

(51) Gordon, M. S.; Freitag, M. A.; Bandyopadhyay, P.; Jensen, J. H.; Kairys, V.; Stevens, W. J. J. Phys. Chem. A 2001, 105, 293.

(52) Ferenczy, G. G.; Rivail, J.-L.; R., S. P.; Náray-Szabó, G. J. Comput. Chem. 1992, 13, 830.

(53) Théry, V.; Rinaldi, D.; Rivail, J.-L.; Maigret, B.; Ferenczy, G. G. J. Comput. Chem. 1994, 15, 269.

(54) Assfeld, X.; Rivail, J.-L. Chem. Phys. Lett. 1996, 263, 100. 
(55) Hohenberg, P.; Kohn, W. Phys. Rev. 1964, 136, B864.

(56) Kohn, W.; Sham, L. J. Phys. Rev. 1965, 140, A1133.

(57) Fiolhais, C., Nogueira, F., Marques, M., Eds. A primer in density functional theory; Lecture notes in physics; Springer: Berlin, 2003.

(58) Kim, M.-C.; Sim, E.; Burke, K. Phys. Rev. Lett. 2013, 111, 073003.

(59) Kim, M.-C.; Sim, E.; Burke, K. J. Chem. Phys. 2014, 140, 18A528.

(60) Gerratt, J.; Mills, I. M. J. Chem. Phys. 1967, $49,1719$.

(61) Handy, N. C.; Schaefer, H. F., III J. Chem. Phys. 1984, 81, 5031.

(62) Pulay, P. WIREs Comput. Mol. Sci. 2013, 4, 169.

(63) Plimpton, S. J. J. Comput. Phys. 1995, 117, 1.

(64) http:// lammps.sandia.gov.

(65) Aradi, B.; Hourahine, B.; Frauenheim, T. $J$. Phys. Chem. A 2007, 111, 5678.

(66) Rappé, A. K.; Goddard, W. A., III J. Phys. Chem. 1991, 95, 3358.

(67) Eichler, U.; Kölmel, C. M.; Sauer, J. J. Comput. Chem. 1996, 18, 463.

(68) Pezeshki, S.; Davis, C.; Heyden, A.; Lin, H. J. Chem. Theory Comput. 2014, 10, 4765.

(69) Boereboom, J. M.; Potestio, R.; Donadio, D.; Bulo, R. E. J. Chem. Theory Comput. 2016, 12,3441 . 